\title{
Review
}

Acta Cytologica 2015;59:2-16

Received: December 23, 2014

DOI: $10.1159 / 000377697$

Accepted: December 29, 2014

Published online: March 24, 2015

\section{Guidelines for the Cytopathologic Diagnosis of Epithelioid and Mixed-Type Malignant Mesothelioma}

\author{
Complementary Statement from the International Mesothelioma Interest Group, Also \\ Endorsed by the International Academy of Cytology and the Papanicolaou Society of \\ Cytopathology
}

\author{
Anders Hjerpe $^{\text {a Valeria Ascoli }}{ }^{\text {b Carlos W.M. Bedrossian }}{ }^{c}$ Mathilde E. Boon ${ }^{d}$ \\ Jenette Creaney ${ }^{\mathrm{e}}$ Ben Davidson $^{\mathrm{f}}$ Annika Dejmek $^{\mathrm{g}}$ Katalin Dobra $^{\mathrm{a}}$ \\ Ambrogio Fassina $^{\mathrm{h}}$ Andrew Fieldi ${ }^{\mathrm{i}}$ Pinar Firat ${ }^{\mathrm{j}}$ Toshiaki Kamei $^{\mathrm{k}}$

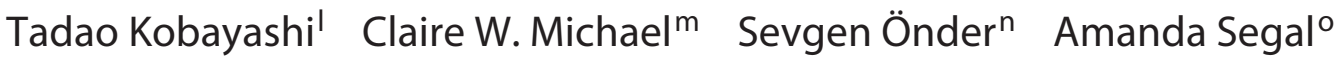 \\ Philippe Vielh ${ }^{p}$
}

\begin{abstract}
${ }^{a}$ Division of Clinical Pathology/Cytology, Department of Laboratory Medicine, Karolinska Institutet, Karolinska University Hospital Huddinge, Huddinge, Sweden; ${ }^{b}$ Department of Radiological, Oncological and Pathological Sciences, Sapienza University, Rome, Italy; ${ }^{\mathrm{C}}$ Rush University Medical College, Chicago, III., USA; ${ }^{\mathrm{d}}$ Leiden Cytology

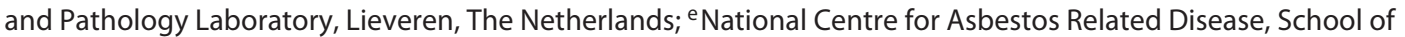
Medicine and Pharmacology, University of Western Australia, QEIl Medical Centre, Perth, W.A., Australia; fDepartment of Pathology, The Norwegian Radium Hospital, Oslo University Hospital, Oslo, Norway; ${ }^{9}$ Department of Laboratory Medicine in Malmö, Lund University, Malmö University Hospital, Malmö, Sweden; h Department of Medicine, University of Padova, Padova, Italy; 'Department of Anatomical Pathology, St Vincents Hospital, Sydney, N.S.W., Australia; J Istanbul University, Istanbul Faculty of Medicine, Istanbul, Turkey; kDivision of Pathology, Yamaguchi Grand Medical Center, Hofu, and 'Cancer Education and Research Center, Osaka University Graduate School of Medicine, Osaka, Japan; ${ }^{\mathrm{m} C a s e}$ Western Reserve University/University Hospitals Case Medical Center, Department of Pathology, Cleveland, Ohio, USA; ${ }^{n}$ Hacettepe University, Faculty of Medicine, Department of Pathology, Ankara, Turkey; ${ }^{\circ}$ Department of Tissue Pathology, PathWest Laboratory Medicine WA, QE2 Medical Centre, Perth, W.A., Australia; ${ }^{\text {PD }}$ epartment of Biopathology, Gustave Roussy Comprehensive Cancer Center, Villejuif, France
\end{abstract}

\section{Key Words}

Malignant mesothelioma · Guidelines - International Mesothelioma Interest Group · Cytology · Ancillary testing

This article is being simultaneously published in Diagnostic Cytopathology (DOI: 10.1002/dc.23271) and Cytopathology (DOI: 10.1111/ cyt.12250). (c) 2015 S. Karger AG, Basel

$0001-5547 / 15 / 0591-0002 \$ 39.50 / 0$

\section{Abstract}

Objective: To provide practical guidelines for the cytopathologic diagnosis of malignant mesothelioma. Data Sources: Cytopathologists with an interest in the field involved in the International Mesothelioma Interest Group (IMIG) and the International Academy of Cytology (IAC) contributed to this update. Reference material includes peer-reviewed publications and textbooks. Rationale: This article is the result of discussions during and after the IMIG 2012 conference in

\section{KARGER 125}

E-Mail karger@karger.com www.karger.com/acy
Correspondence to: Prof. Anders Hjerpe

Division of Clinical Pathology/Cytology, Department of Laboratory Medicine Karolinska Institutet, Karolinska University Hospital Huddinge SE-14186 Huddinge (Sweden)

E-Mail anders.hjerpe@ karolinska.se 
Boston, followed by thorough discussions during the 2013 IAC meeting in Paris. Additional contributions have been obtained from cytopathologists and scientists who could not attend these meetings, with final discussions and input during the IMIG 2014 conference in Cape Town.

(C) 2015 S. Karger AG, Basel

\section{Introduction}

During the previous International Mesothelioma Interest Group (IMIG) biennial meetings thorough discussions have resulted in published guidelines for the pathologic diagnosis of malignant mesothelioma (MM). However, previous recommendations have stated that the diagnosis of MM should be based on histological material only $[1,2]$. Accumulating evidence now indicates that the cytological diagnosis of MM supported by ancillary techniques is as reliable as that based on histopathology, although the sensitivity with cytology may be somewhat lower [3-5]. Recognizing that noninvasive diagnostic modalities benefit both the patient and the health system, future recommendations should include cytology as an accepted method for the diagnosis of this malignancy [6, 7]. This article describes the consensus of opinions of the authors on how cytology together with ancillary testing can be used to establish a reliable diagnosis of MM.

The diagnosis of MM can in the majority of cases be based on the evaluation of the effusion [8], and diagnostic criteria are readily available and illustrated in text books [9-12]. In such cases, the morbidity of the patient is significantly decreased since a definitive cytological diagnosis negates the need for more invasive diagnostic procedures, and eliminates the necessity for a biopsy, which has a higher potential for morbidity and a documented increased risk of tumor seeding [13, 14]. Definitive treatment can also be immediately initiated, avoiding delay. However, when effusion cytology is inconclusive for the diagnosis of MM, tissue core biopsy should be performed as previously recommended. The two techniques are complementary.

It should be emphasized that the important measure here is the reliability of the cytological diagnosis, that is, the positive predictive value, which has been shown to be equal to that of histopathology for use in both clinical and medico-legal contexts. Although a history of exposure to asbestos can be significant and reinforces the suspicion for MM, it is not mandatory for the diagnosis. Nor can the diagnosis of MM rely on the location of the tumor in the pleura or peritoneum, or on the gender of the patient.
The diagnosis of MM on cytology includes two steps: the establishment that the effusion is malignant, and then diagnosis of the mesothelial origin of the malignant cells. Cytopathology is rightfully recognized as a subspecialty of pathology in its own right and the accuracy of the procedure for the diagnosis of cancer on cytological grounds in experienced hands carries the same weight as that of a tissue biopsy. Proficiency in cytological diagnosis can be achieved through knowledge, training and experience obtained by fellowship programs and daily practice. With such training in cytopathology, the experienced cytopathologists can often recognize MM based on a routinely stained specimen [4]. The accurate diagnosis should, however, always be verified by the use of ancillary techniques. Immunocytochemistry (ICC) or immunohistochemistry (IHC) will in most cases be sufficient, while other techniques such as molecular biology, electron microscopy and biomarker analyses may add further support and even improve sensitivity. Because of the medicolegal ramifications, and with the availability of many mesothelial and adenocarcinoma markers, we strongly recommend that all cases should be confirmed with ICC/ IHC.

An MM diagnosis can in the majority of cases be achieved based on cytomorphology and ancillary testing. The main obstacles for the diagnosis are: (i) the low yield of the diagnostic cells due to hypocellularity, bleeding or inflammation; (ii) a cytopathologist's lack of experience, and (iii) lack of awareness of this diagnostic possibility. A low yield of diagnostic cells can be circumvented by concentration techniques, such as liquid-based cytology and the cell block technique [15].

Frequently, the diagnosis of MM can be established on the first effusion, making a follow-up biopsy redundant, particularly in inoperable cases where adjuvant therapy can be initiated. However, a negative finding does not exclude this diagnosis. This is especially common when the tumor is dominated by a sarcomatoid component, and core biopsy is indicated in these cases in line with the previous guidelines, particularly when surgery is considered, because the presence of a sarcomatoid component may influence the therapeutic management [14].

\section{How to Handle the Material}

The effusion is preferably sent to the laboratory fresh if possible with anticoagulants (heparin EDTA or sodium citrate) present, but without added fixatives and it should be refrigerated at $4^{\circ} \mathrm{C}$ until processing. When longer 
Table 1. Cytomorphological criteria indicating malignancy

The presence of numerous large tissue fragments and balls of cells is diagnostic of malignancy in pleural fluid cytology (fig. 1)

The presence of overtly malignant cells, either as single cells or in tissue fragments, where the degree of nuclear abnormality is diagnostic of a malignant process

transportation times are needed, a volume of $50 \%$ ethanol can be added as preservative.

Upon arrival in the laboratory, the fluid should be processed without delay. Refrigerated samples should be brought to room temperature, particularly when using preparation techniques associated with liquid-based cytology. To prepare a cell pellet the material is centrifuged at $1,000 \mathrm{~g}$ or more for $10 \mathrm{~min}$. For cytomorphological evaluation it is recommended that both air-dried and wet-fixed smears or cytospins are prepared to be stained with Papanicolaou (Pap), and either Diff-Quick or MayGrünwald-Giemsa (MGG). Cytospins or cell blocks are prepared for ICC or IHC, respectively. When effusions are bloody, hemolysis should be attempted prior to slide preparation.

Where electron microscopy is available, an aliquot of the cell pellet should be transferred to buffered glutaraldehyde without delay. The cells can then be kept at $4^{\circ} \mathrm{C}$ for later embedding if the case subsequently requires this. A 2- to 5-ml aliquot of the cell-free supernatant can be stored at $-20^{\circ} \mathrm{C}$ for later biomarker analyses, if these are available. Electron microscopy can sometimes also be applied to paraffin-embedded tissue, when a fresh specimen is not available and the diagnosis of mesothelioma depends solely on the existence of archival material [16].

\section{Basic Cytomorphology}

Serous effusions from patients with MM at any location can be divided into three groups:

- Those cases that are malignant on cytomorphological criteria;

- Those cases where the cytological material requires some form of ancillary testing to establish malignancy;

- Those cases that cannot be diagnosed by cytology this category includes cases with minimal cell shedding, typically almost all sarcomatoid and desmoplastic MM, and also those epithelioid MM that do not
Table 2. Summary of cytomorphological criteria indicating malignant mesothelioma (see fig. 1-3)

Highly cellular sample, often including large and small tissue fragments

Mesothelial cells which are significantly larger than normal mesothelial cells either singly or in tissue fragments; each of the components of the whole cell is enlarged: cytoplasm, nucleus and nucleolus

Papillary tissue fragments forming spheres with a smooth surface or berry-like tissue fragments with a scalloped surface, sometimes with clear spaces or 'windows' between the cells

Acidophilic extracellular matrix cores, also known as collagen or basement membrane cores, within the tissue fragments and an extracellular granular acidophilic background indicating large amounts of hyaluronan

The presence of macro nucleoli

Protrusions from the cell membrane or blebbing

Prominent degree of cell-within-cell arrangements

Background with multinucleated giant cells and small pyknotic eosinophilic or orangeophilic cells

Vacuoles overlapping the nuclei of MGG-stained cells

have malignant cytomorphology or diagnostic positive ancillary tests.

In addition to cytomorphology, the first two categories generally require ancillary techniques such as ICC or $\mathrm{IHC}$, and in rare cases electron microscopy, to confirm that the phenotype is of mesothelial origin. The main differential diagnosis in this setting is usually between MM and metastatic adenocarcinoma. The third category will require some form of histological material.

\section{Cytomorphological Features of Malignancy in}

Effusions

The first basic question in evaluating the cytomorphology of pleural effusions is whether the cells are malignant or not. A malignant condition can in many cases be diagnosed based on routinely stained cell preparations (table 1).

Overtly malignant cells with diagnostic nuclear malignant features may be seen in various anaplastic tumors, and in this setting establishing the phenotype may be difficult. Such cells are less common in MM than in adenocarcinoma and other metastatic malignancies. Attempts to establish a malignant diagnosis should be undertaken when the sample contains exceptionally large numbers of
Hjerpe et al. 
Fig. 1. Effusions with epithelioid mesothelioma. The specimen is often highly cellular, containing large and small tissue fragments. a Pap stain. b MGG stain. Scale bar $=50 \mu \mathrm{m}$.

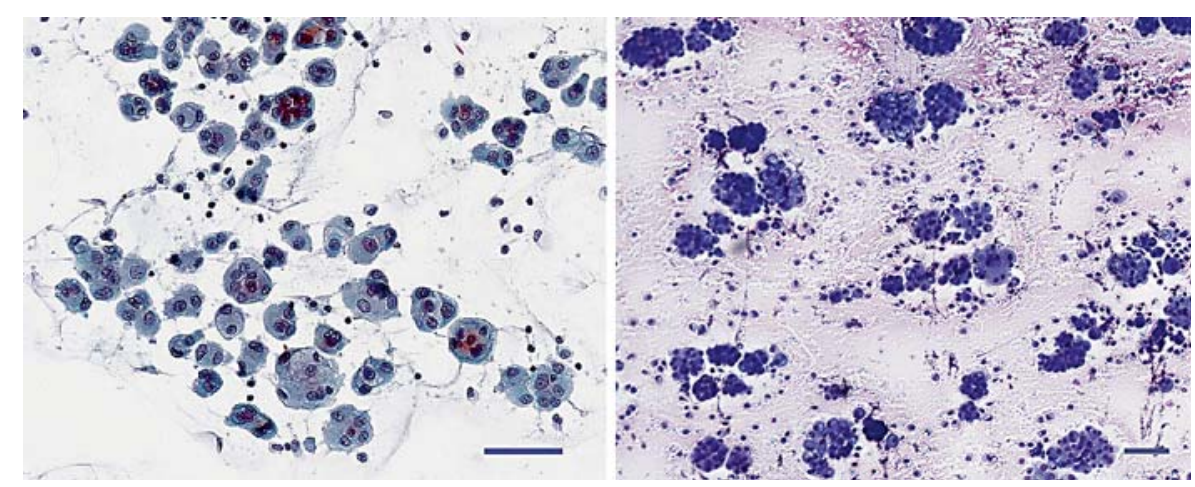

mesothelial cells even when the nuclear atypia is less apparent. Fluorescent in situ hybridization (FISH) and/or ICC or IHC will often confirm the presence of malignant cells.

\section{Cytomorphological Features Suggesting MM, but}

Requiring Some Form of Ancillary Confirmation

The main cytologic findings of MM in effusions have been well described in the literature $[8,17]$, and the criteria have recently been reevaluated [18]. The experienced cytopathologist will in many cases recognize the MM effusion, but the definite diagnosis should be supported by ancillary techniques. It is the experience of several laboratories of the authors that the diagnosis is completely reliable when this approach is used.

At low power, the first indication of MM is often a highly cellular slide with numerous tissue fragments (table 2). Although pleomorphic, the cells appear monotonous at scanning magnification and there is a lack of a second 'alien' cell population. It should be remembered that some cases are extremely discohesive [19], and high cellularity should be enough to motivate further ancillary tests to diagnose a possible MM. These cellular cases, with or without papillary tissue fragments, are sometimes difficult to distinguish from reactive mesothelial hyperplasia, which in the past has been called 'mesotheliosis'. On the other hand, in less differentiated MM the nuclear atypia is evident and the malignancy is diagnosed on nuclear criteria of malignancy, while the mesothelial phenotype can be less obvious. A suggestive finding that establishes the mesothelial origin is the occurrence of intercellular clear slits or 'windows' between the cells in the tissue fragments. Minute tissue fragments can show cell-withincell arrangements known previously as 'cell cannibalism' or cellular clasping and pinching (fig. 1-3).

Examining the cytoplasmic periphery and the cell membrane can also shed light on the mesothelial origin of cells. In well-fixed, Pap-stained slides, a brush border can often be detected corresponding to the microvilli seen on electron microscopy, and there is differential staining between the central and peripheral cytoplasm; that is, there is endoectoplasmic demarcation or two-tone staining. However, this finding can be less obvious in liquidbased cytology preparations. In MGG-stained MM cells a diffuse pinkish haze close to the cell border is frequently noted (fig. 3). This may represent hyaluronan, which is attached during its synthesis in the cell membrane and therefore not extracted during staining. 'Blebbing' is also a common finding in MM cells, with rounded cytoplasmic protrusions extending from the plasma membrane. The MM cytoplasm often contains glycogen deposits, which manifest as elongated yellowish vacuoles or deposits beneath the cell membrane and are best seen in Papstained slides.

Background features may also raise the suspicion of MM. The MGG stain often shows a red or pink granular background, a finding that correlates with high concentrations of hyaluronan in the effusion (fig. 2). Pink extracellular material can also be found in pockets between cells in papillary tissue fragments in the MGG. This water-soluble glycosaminoglycan is extracted and, thus, not seen in the Pap-stained material.

Another cytoplasmic feature indicative of MM is the presence of numerous small lipid-containing vacuoles (fig. 3). They can be seen in the entire cytoplasm of MGGstained, air-dried preparations and, when overlapping the nucleus, they appear as 'punched out holes'. These vacuoles represent a degenerative process and increase with time if the effusion is kept unfixed [20,21]. These droplets are extracted by ethanol fixation for the Pap stain, but they are easily demonstrated in MGG-stained slides. 
Fig. 2. Cytomorphology of epithelioid mesothelioma cells and tissue fragments in effusions. The tissue fragments sometimes show gaps or windows (a, b), and occur both as spheres with smooth surfaces $(\mathbf{c}, \mathbf{d})$ and in berry-like clusters with scalloped surfaces $(\mathbf{e}, \mathbf{f})$. The tissue fragments may contain acidophilic extracellular matrix cores, also known as collagen or basement membrane cores $(\mathbf{g}, \mathbf{h})$, which with MGG become strongly acidophilic, similar to the extracellular granular material that can be seen in the background (see also $\mathbf{f}$ and fig. 1), indicating large amounts of hyaluronan. a, c, e, g Pap stain. b, d, f, h MGG stain. Scale bar $=50 \mu \mathrm{m}$.

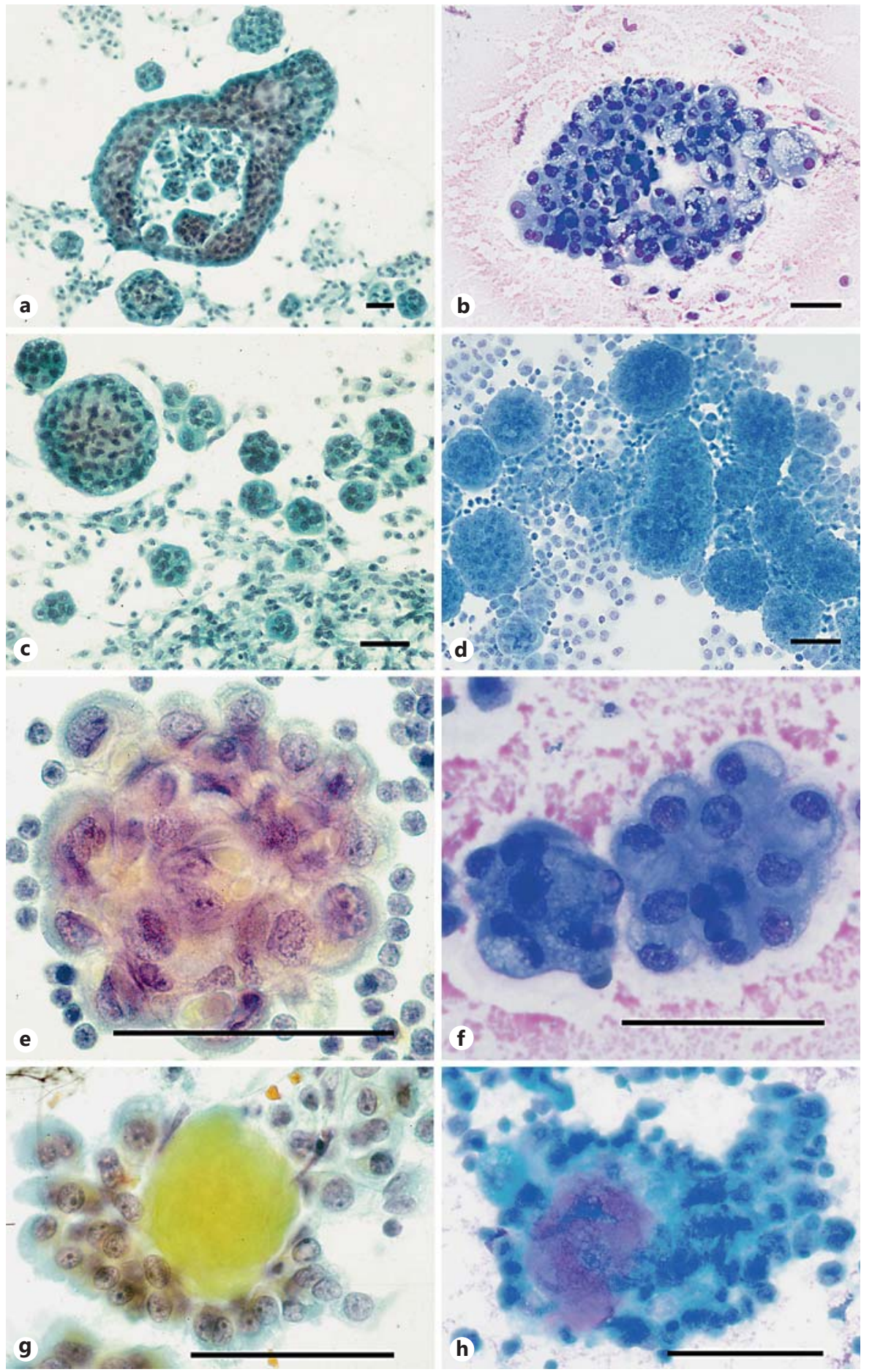

Acta Cytologica 2015;59:2-16

DOI: $10.1159 / 000377697$
Hjerpe et al. 


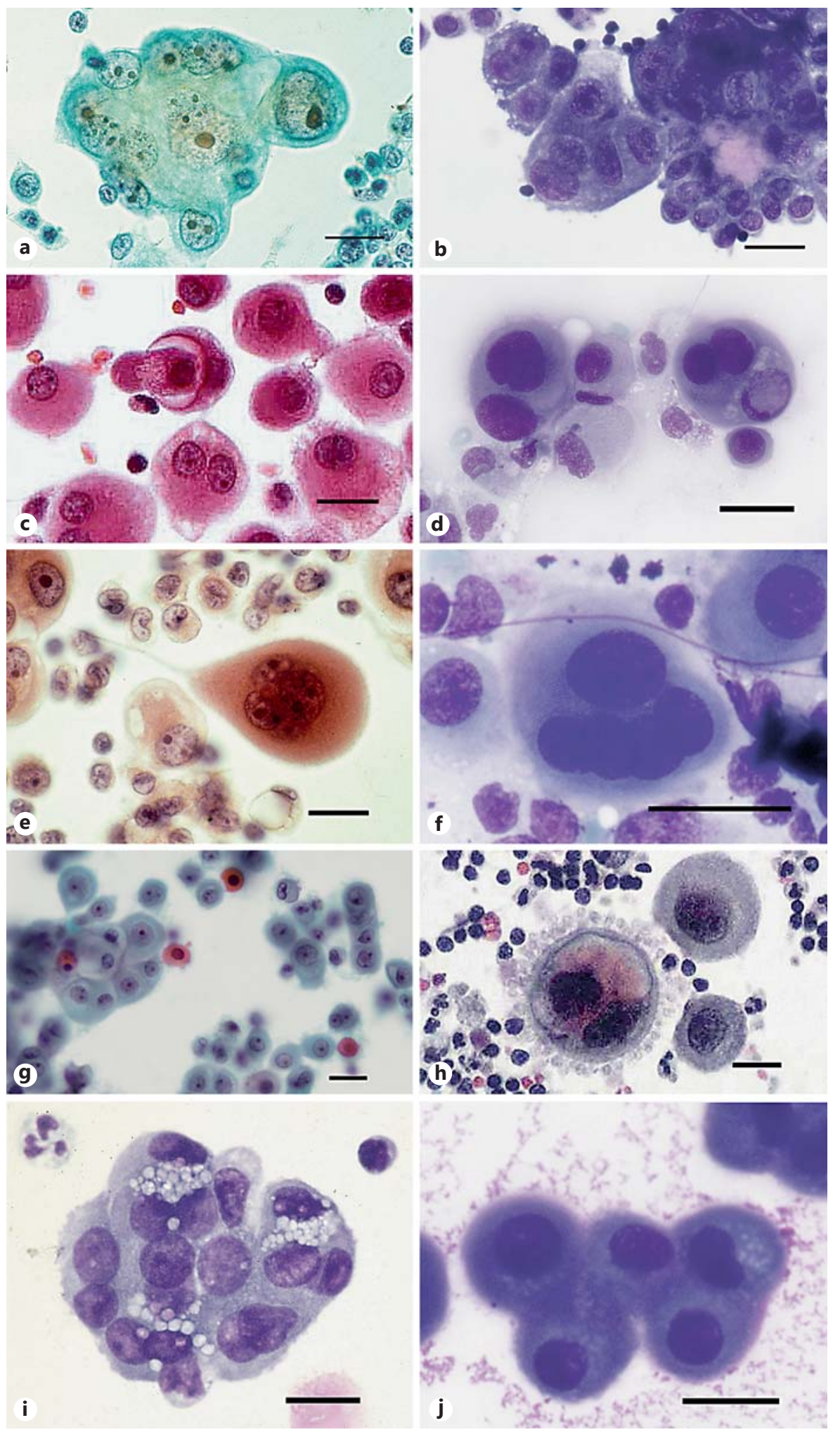

Fig. 3. Cytomorphology of the epithelioid mesothelioma cells in effusions. The MM cells often show macro nucleoli $(\mathbf{a}, \mathbf{b})$. There is a prominent degree of cell-withincell arrangements $(\mathbf{c}, \mathbf{d})$ and the tumor cells are sometimes multinucleated $(\mathbf{e}, \mathbf{f})$. Some cells may be squamoid with eosinophilic or orangeophilic cytoplasm and pyknotic nuclei $(\mathbf{g})$, while others show rounded protrusions or 'blebbing' from the cell membrane (h). The tumor cells may develop cytoplasmic vacuoles which punch holes in the nucleus in MGG-stained slides (i) as well as a reddish haze at the periphery of the cells $(\mathbf{j})$, corresponding to the location of hyaluronan synthesis. a, c, e, f Pap stain. b, d, f, $\mathbf{h}-\mathbf{j}$ MGG stain. Scale bar $=25 \mu \mathrm{m}$.
IMIG Guidelines for the Pathologic Diagnosis of MM
Acta Cytologica 2015;59:2-16 DOI: $10.1159 / 000377697$ 


\section{ICC and IHC}

The main differential diagnoses of MM are adenocarcinoma and reactive mesothelial proliferations, so-called 'mesotheliosis', and the diagnosis in the majority of cases can be resolved using ICC on cytospins or IHC on cell blocks. Ancillary testing must be available in laboratories diagnosing MM based on effusions.

\section{Mesothelial Phenotype of Tumor Cells}

It is recommended that a panel of at least four antibodies should be used, two in favor and two against MM. The diagnosis should never be based on one single ICC or IHC reaction. Numerous antibodies for MM are commercially available, but most are not entirely specific and may show cross reactivity with other tumors, such as ovarian carcinomas.

The antibodies and epitopes used for cytological samples are the same as those recommended for formalinfixed histological material $[1,2]$. In cell blocks, as well as in cytospin preparations, epithelial membrane antigen (EMA) with accentuated reactivity at the cell membrane is often used to support the diagnosis of MM [22]. However, EMA is not a specific mesothelial marker, and the typical cell membrane reactivity may also be seen in adenocarcinomas. Other antibodies supporting the diagnosis of an MM are calretinin, podoplanin (D2-40), WT1, CK5/6 and mesothelin. Calretinin can, however, also be expressed in breast carcinomas [23], and CK5/6 and mesothelin are sometimes also expressed in ovarian and pancreatic adenocarcinoma (fig. 4).

Recommended antibodies for excluding a mesothelial origin include CEA, BerEp4 and MOC-31, TTF-1, CD15 and Sialyl-TN. Among the different anti-CEA antibodies available to exclude an MM, the best-performing reagent seems to be a monoclonal preparation (No. A0115, DAKO) [24]. Although reactivity to the DAKO monoclonal CEA antibody is rarely, if ever, seen in MM, a proportion of MM will express BerEp4 or MOC-31. Double staining with calretinin and BerEp4 will help in most MM cases, as the calretinin reactivity will override that of BerEp4 (fig. 4, 5).

In summary, to demonstrate the mesothelial origin of a tumor cell population, it is recommended that two of the above antibodies in favor of MM and two excluding the diagnosis be used in a panel: EMA (membranous staining pattern) and calretinin, CEA and BerEp4 will show typical reactivity for MM (the first two positive and the second two negative) in most cases, and will exclude adenocarcinomas (the first two negative and the second two positive) [25]. If the results are equivocal, additional MM-positive antibodies, such as podoplanin and WT1, may be useful provided the adenocarcinoma markers are negative. Also, additional MM-negative antibodies, such as CD15 and/or B72.3, can be considered. If there is a known history of another malignancy such as breast, intestinal or lung carcinomas, it is worth including specific markers for such malignancies, such as estrogen receptor or GATA3, CDX2 and TTF1, respectively.

\section{MM versus Reactive Mesothelium ('Mesotheliosis')}

It is more difficult to distinguish reactive mesothelial cells from malignant ones based on IHC or ICC, and at least two antibodies should be used in a panel. Benign mesothelial cells are known to contain muscle filaments and they express desmin. The mesothelial cell loses this reactivity to desmin early during the oncogenic process, and the absence of such immunoreactivity is a commonly recommended criterion for MM. In the same way, the typical cell membrane reactivity to EMA in the mesothelial cell is a strong indicator of malignancy, but the specificity for MM may vary between different anti-EMA antibodies. Data indicate that the E29 clone (DAKO) has superior performance in distinguishing malignant from reactive mesothelial cells [26]. This is even better seen when performed as a double stain for desmin together with EMA (fig. 5). A similar use has also been described with antibodies against CD146, Imp3 and Glut-1 [27-29]. Furthermore, a general accumulation of p53 in cell tumor nuclei can in some cases be used as further supporting evidence of malignancy [30].

\section{Peritoneal and Pericardial MM}

Since the majority of MM occurs in the pleural cavity, most diagnostic methods have been validated on pleural effusions. MM may also arise in the other serous cavities, where they can show a different clinical spectrum indicating biological differences. Peritoneal MM generally affects younger patients and has a comparable incidence among women and men. A particular papillary neoplasm with superficial spread and bland cytology is designated

Fig. 4. Immunohistochemical and immunocytochemical reactivities of MM cells as seen in cell blocks (1st and 3rd column) and cytospin preparations (2nd and 4th column). EMA and D2-40 positivity is accentuated at the cell membrane, while specific WT1 reactivity is nuclear. The effusion with MM cells often also contains scattered benign mesothelial cells that contain desmin reactivity. Scale bar $=50 \mu \mathrm{m}$.

(For figure see next page.)
Hjerpe et al. 


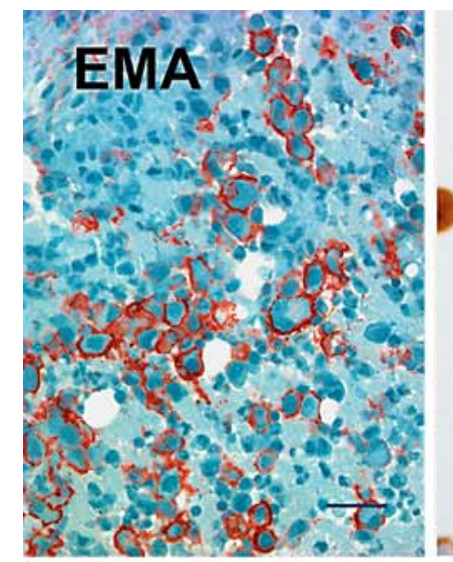

\section{WTI}
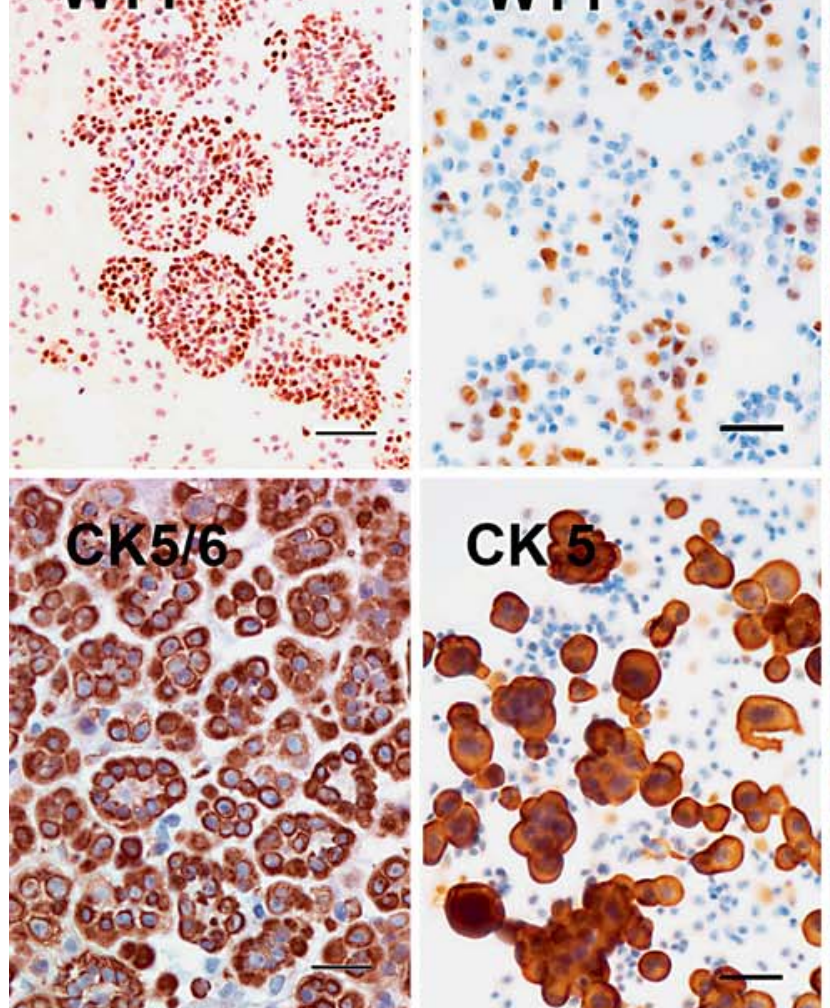

WT1
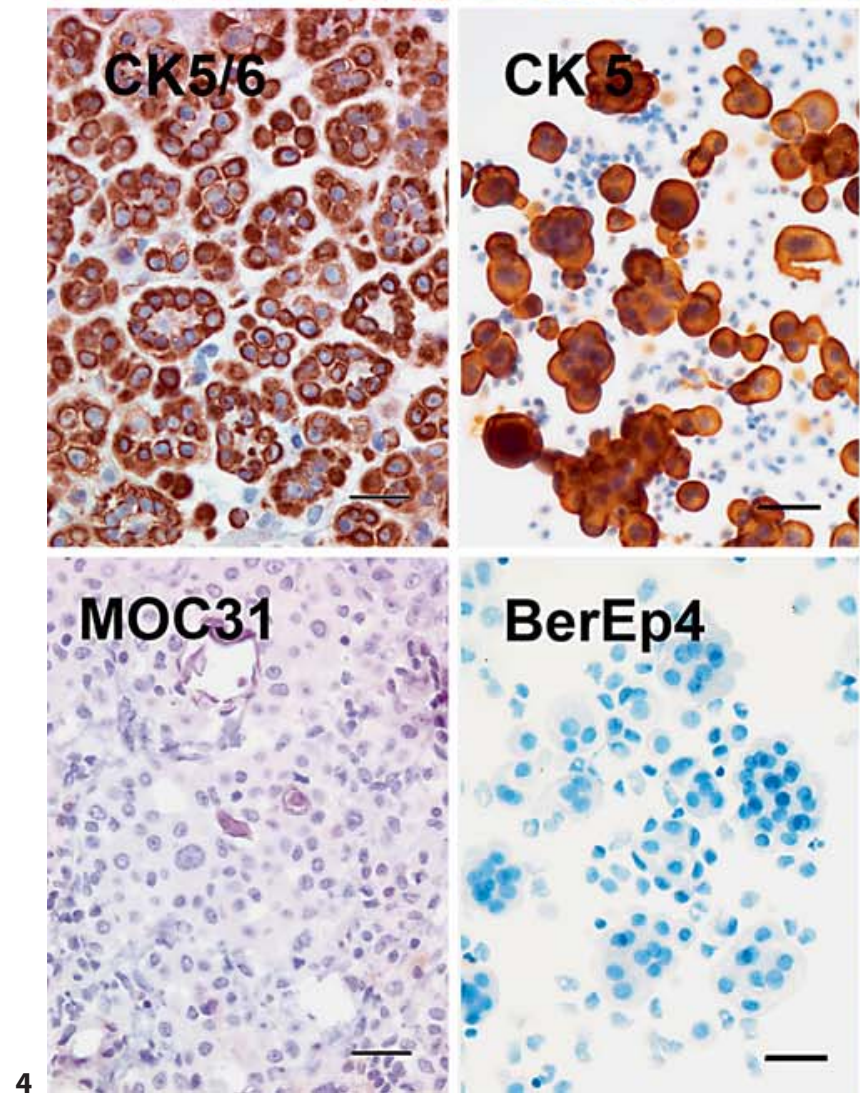

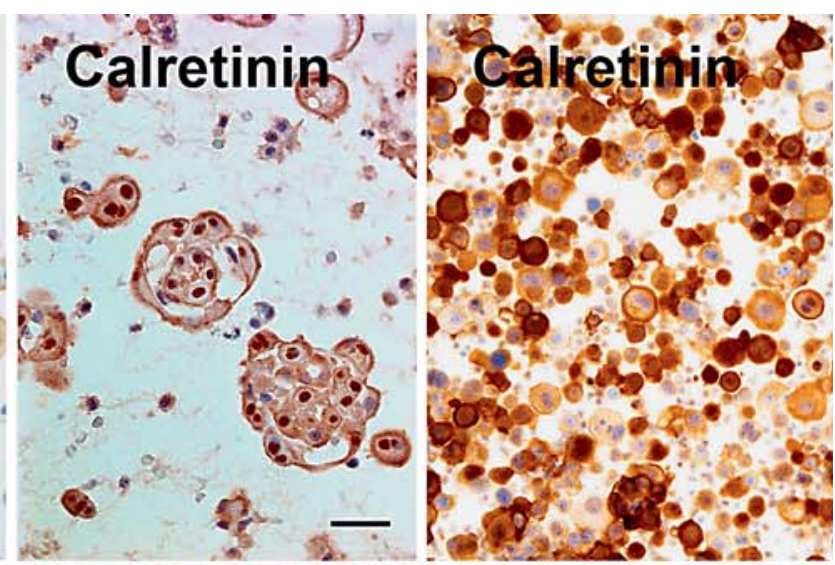

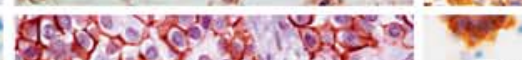

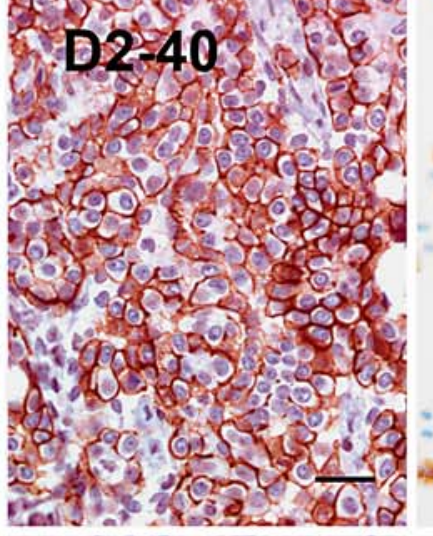

D2-40

Desmiñn Desmin
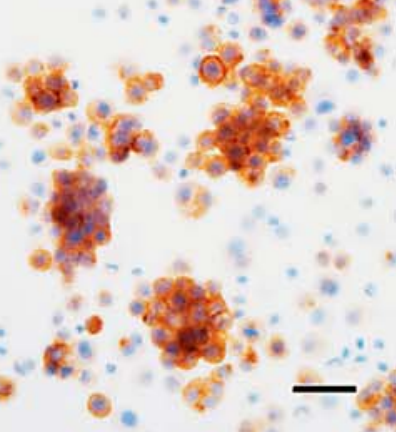
Fig. 5. The use of double staining. Cytospin preparations double stained for BerEp4 (brown) and calretinin (red; a, c) and desmin (brown) and EMA (red; b, d) are useful to distinguish MM from both adenocarcinomas and reactive mesothelial cell proliferations. In MM samples the tumor cells show dominant reactivity to calretinin and EMA (a, b), while reactive benign mesothelial cells react to calretinin and desmin (c, d). Scale bar $=100 \mu \mathrm{m}$.

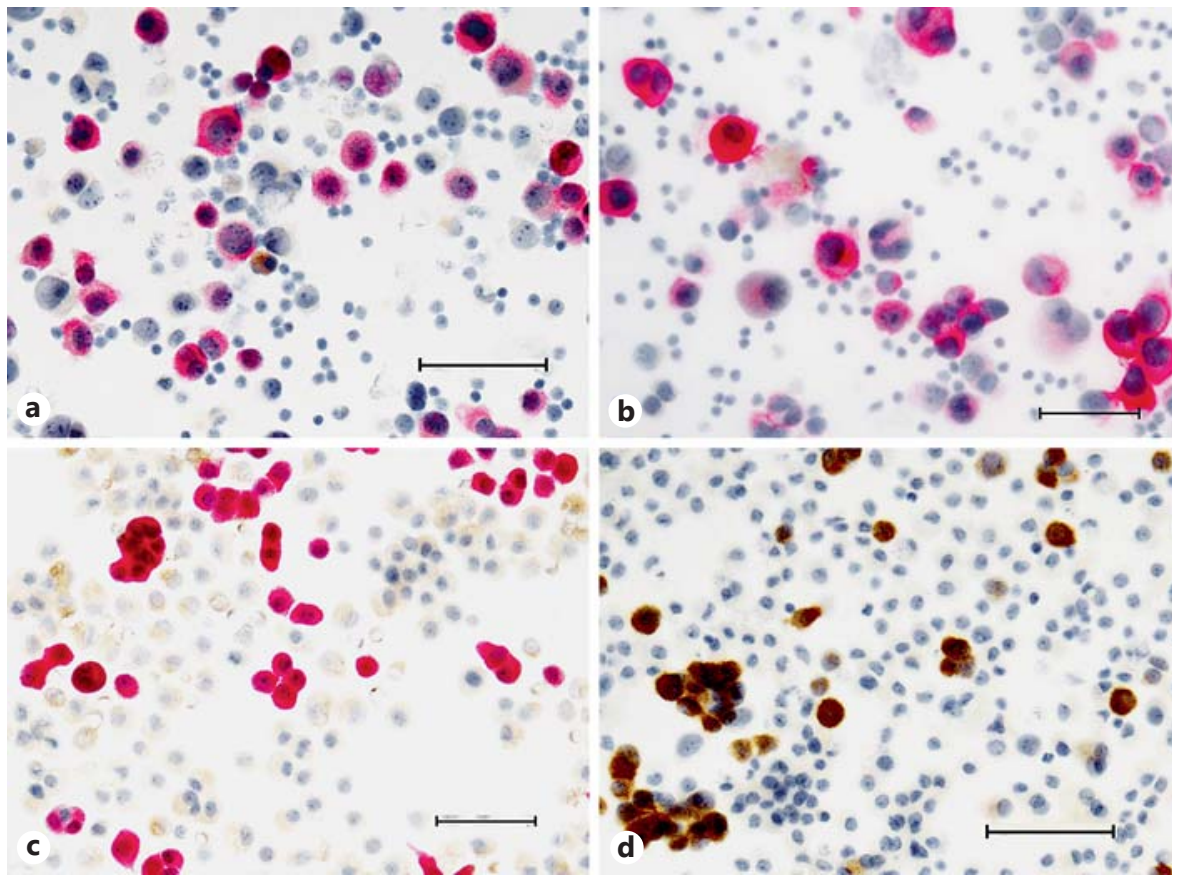

'well-differentiated papillary mesothelioma' [31] and usually occurs in the peritoneal cavity of women as multifocal lesions [32].

The challenging differential diagnosis in the peritoneum is between MM and ovarian or peritoneal serous carcinoma [33]. These tumors have a common histogenesis, may be difficult to differentiate morphologically and coexpress many of the diagnostic markers discussed in the previous section. However, the serous carcinomas of ovary, uterus and peritoneal origin stain positive for BerEp4, PAX8, B72.3, BG8, CA 19-9, Leu-M1 (CD15), MUC4 and estrogen receptor, all of which are rarely or never expressed in MM [20, 33, 34] (fig. 6).

Pericardial MM are less common. The diagnosis is based on the same criteria as pleural and peritoneal MM, but pericardial fluids are often associated with inflammatory conditions, and here the challenge is to distinguish the MM from mesothelial reactive changes. Malignancy must in these cases be established and verified with caution, using ICC, IHC and possibly other techniques, such as analysis of ploidy by FISH (see below).

\section{Optional Ancillary Techniques}

The diagnostic sensitivity of MM in effusions can be improved further by the use of other ancillary techniques, such as molecular biology, electron microscopy and chemical analyses of soluble biomarkers in the effusion supernatant. Equipment for electron microscopy is not generally available, while other techniques such as ELISA and FISH are more widely available.

\section{Analysis of Soluble Biomarkers}

Tumor-specific markers can be demonstrated by ICC or IHC in the tumor cell, and are also found in the effusion supernatant, either as secretory products or as a result of tumor cell decay. These tests can be run in parallel with the morphologic evaluations on all effusions with malignancy of unknown origin, those suspicious for malignancy and those with plentiful mesothelial cells.

The two best-established biomarkers are hyaluronan and mesothelin [35-40]. Additional possible MM biomarkers have been reported in the recent literature [4143], and are awaiting further evaluation and validation for clinical practice. Hyaluronan is a glycosaminoglycan often secreted in large amounts into MM effusions: a concentration exceeding $250 \mathrm{~g} / \mathrm{l}$ is only seen in MM effusions [40, 44 ] and appears to be specific for this diagnosis, but such high levels are only obtained in 50-60\% of MM effusions.

The mesothelin family of proteins is one of the most investigated soluble biomarkers to aid MM diagnosis, both in sera and effusions. The protein is cleaved into a C-terminal $\sim 40-\mathrm{kDa}$ protein, which is commonly referred to as 'mesothelin' or C-ERC/mesothelin, while the solubilized, $\mathrm{N}$-terminal, $30-\mathrm{kDa}$ part is referred to as 


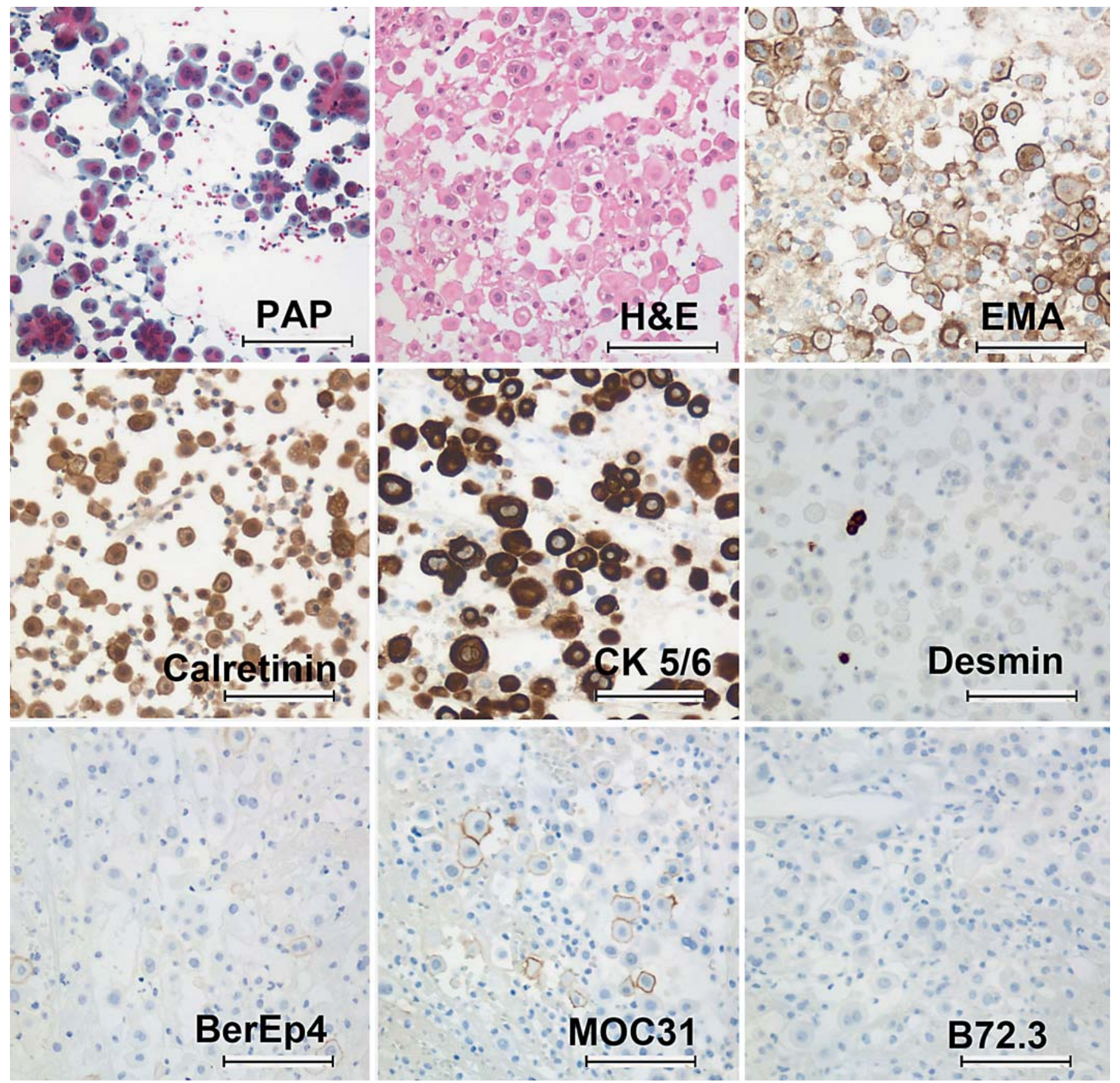

Fig. 6. Diagnostic markers used in the differential diagnosis between DMPM and serous carcinoma. A large number of tumor cells is seen in Pap-stained slides and a HE-stained section from a cell block from a DMPM effusion. DMPM expresses EMA, cal- retinin and CK5/6 and, unlike reactive mesothelial cells, it only has focal desmin expression. Ber-EP4 and MOC-31 stain only a few cells, whereas the B72.3 stain is negative. Scale bar $=50 \mu \mathrm{m}$. 
'megakaryocyte potentiating factor' or N-ERC/mesothelin. Both these fragments can be analyzed in the effusion supernatant, and high levels indicate malignancy with sensitivity similar to that of hyaluronan.

The analysis of hyaluronan and mesothelin can be performed in two steps, with the second analysis only performed when the first marker shows moderately elevated values. The high specificity of hyaluronan for MM makes this glycosaminoglycan the best choice for the first analysis. Concentrations exceeding $250 \mathrm{mg} / \mathrm{l}$ indicate an MM, while samples with $60-249 \mathrm{mg} / \mathrm{l}$ can be analyzed for their concentration of mesothelin. A value exceeding 20 $\mathrm{nM}(0.8 \mathrm{mg} / \mathrm{l})$ of C-ERK/mesothelin or $50 \mathrm{nM}(1.5 \mathrm{mg} / \mathrm{l})$ of $\mathrm{N}-\mathrm{ERK} /$ mesothelin likewise will indicate the MM diagnosis, although this marker can be produced by carcinomas, particularly ovarian and pancreatic carcinomas, and is therefore not entirely specific. Intermediate values can be applied to a logistic interpretation model to support the diagnosis of MM in additional cases [40]. The levels of hyaluronan and mesothelin correlate and tend to identify the same cases, but this is not absolute, and the combined use in a logistic battery will improve the sensitivity.

\section{Electron Microscopy}

When available, electron microscopy can be of help when ICC and IHC are not entirely conclusive or when the biomarkers indicate an MM in spite of inconclusive cytomorphology. Effusions are well suited for electron microscopy, provided a portion of the cell pellet is properly fixed early in the process. This requires that an aliquot of the first fresh cell pellet is fixed in glutaraldehyde without delay. The ultrastructural analysis can then be performed on demand.

The ultrastructure of MM cells is well known, and electron microscopy is considered the gold standard for the diagnosis of MM (fig. 7). The finding of apical cell membranes with microvilli at basolateral surfaces, forming 'extracellular neolumina', and/or forming an intracellular vesicle or 'intracellular neolumina' is pathognomonic for a malignant cell (fig. 7c, d). To be certain that the group of cells represent a tissue fragment of the tumor and not just an aggregate of cells, desmosomes should be present between the cells (fig. 7d), and the reliable diagnosis of $\mathrm{MM}$ is best based on cell tissue fragments and not on an entirely dissociated cell population.

Apart from criteria seen in cytomorphology including nuclear pleomorphism and atypical nucleoli, the nuclear morphology of the MM cell in electron microscopy is often highly irregular in a way never seen in the light mi- croscope (fig. 7a). The characteristic feature of the MM cell is the presence of long slender microvilli without any core rootlets and glycocalyx formation on the surface (fig. $7 \mathrm{~d}$ ). These microvilli are distinctly different from those seen in adenocarcinoma, in which the cell villi are coarser with a stiffer rod-like appearance and often with a distinct glycocalyx. Although not as abundant, long slender microvilli may also appear on reactive mesothelial cells, and it is important that the malignant nature of these cells is established by the presence of extra- and intracellular neolumina. Also, the MM cell often contains glycogen deposits, and microtubules are often present as a 'scarf' around the nucleus (fig. 7e, f).

Electron microscopy, when all criteria are demonstrated, clearly distinguishes MM from adenocarcinomas, but a conclusive MM diagnosis can only be expected in some $50 \%$ of the cases; the main limiting factors are the need for well-preserved cellular material and the presence of papillary and other tissue fragments. However, the analysis is useful, particularly when ICC and IHC fail to confirm the diagnosis and molecular testing is not available, and it may also be helpful in diagnosing unusual rare variants such as small-cell, deciduoid and clear-cell variants.

\section{Molecular Biology}

A malignant condition can also be demonstrated by the presence of an aneuploid cell population. This can be performed by FISH analysis, and the use of 4 target sequences, such as in the Abbot UroVysion ${ }^{\circledR}$ kit, are sufficient in most cases [45]. Three probes of this kit label centromeric sequences on chromosomes 3,7 and 17 , thus showing gains or losses of the chromosome, while the fourth probe labels the 9p21 band containing the p16INK gene. Finding a homozygous deletion of the $9 \mathrm{p} 21$ band is virtually always associated with malignancy and is particularly common in certain malignancies, among them MM, while it occurs much less in carcinomas metastatic to the serous cavities (fig. 8). The deletion will therefore support the diagnosis of an MM, although it is not pathognomonic.

A second criterion for malignancy - that of cells with gains in at least two of the probed centromeric sequences - must be interpreted with care, and caution is advised when interpreting tri- or tetraploid signals from benign cells in the S or G2 phase. Demonstration of telomerase activity is a promising general marker of malignancy, and has been shown to assist the diagnosis of MM in cases not resolved by morphology, ICC/IHC or hyaluronan analysis [46]. The best results so far have been achieved using the in situ TRAP assay [47], but this has not yet been established in routine practice.
Hjerpe et al. 
Fig. 7. Electron microscopy of MM in effusions. The nuclear pleomorphism is normally more extensive than seen in cytomorphology (a, b). Typically, the apical surface of MM cells is covered by long, slender microvilli, completely devoid of any glycocalyx, and malignancy is revealed by the finding of neolumina, i.e. apical cell membranes with microvilli as either a cytoplasmic vacuole (c) or in-between cells with connecting desmosomes, replacing the basolateral membrane (d). Cytoplasmic filaments are often seen surrounding the nucleus as a 'scarf (e), sometimes forming coarser tonofibril structures (f; see also fig. $3 g$ ).

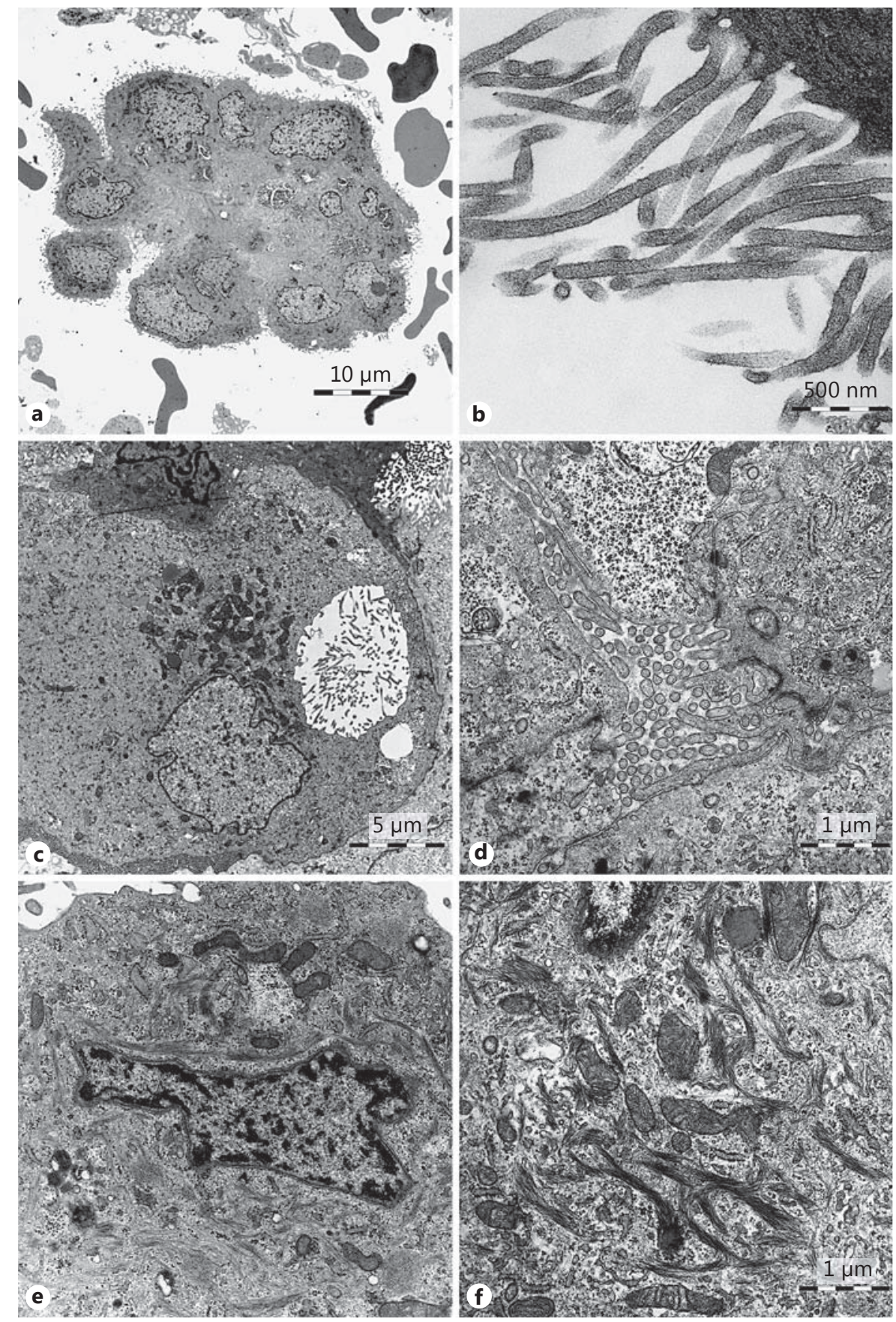

\section{Summary of Differential Diagnoses}

The two main differential diagnoses of MM are adenocarcinoma and benign, reactive mesothelial cells. The distinction of MM from metastatic carcinoma is in most cases made with the help of ICC or IHC. Metastatic adeno- carcinomas react with BerEp4, B72.3, CEA and/or CD15. MMs are positive to calretinin (stronger than to BerEp4), podoplanin (D2-40), mesothelin, EMA accentuated on the cell membrane and WT1, although the last three are also common in ovarian carcinomas. Antibodies indicating a specific primary site greatly assist the diagnosis of 
Fig. 8. FISH analysis of ploidy. The Abbot UroVysion $^{\circledR}$ kit labels centromeric sequences on chromosomes 3 (red), 7 (green) and 17 (blue), showing gains or losses of the chromosome, while the fourth probe (yellow) labels the 9p21 band, containing the p16INK gene. The benign cell (a) shows two signals for each probe while the MM cells often present with homozygous deletion of the 9p21 band (b).
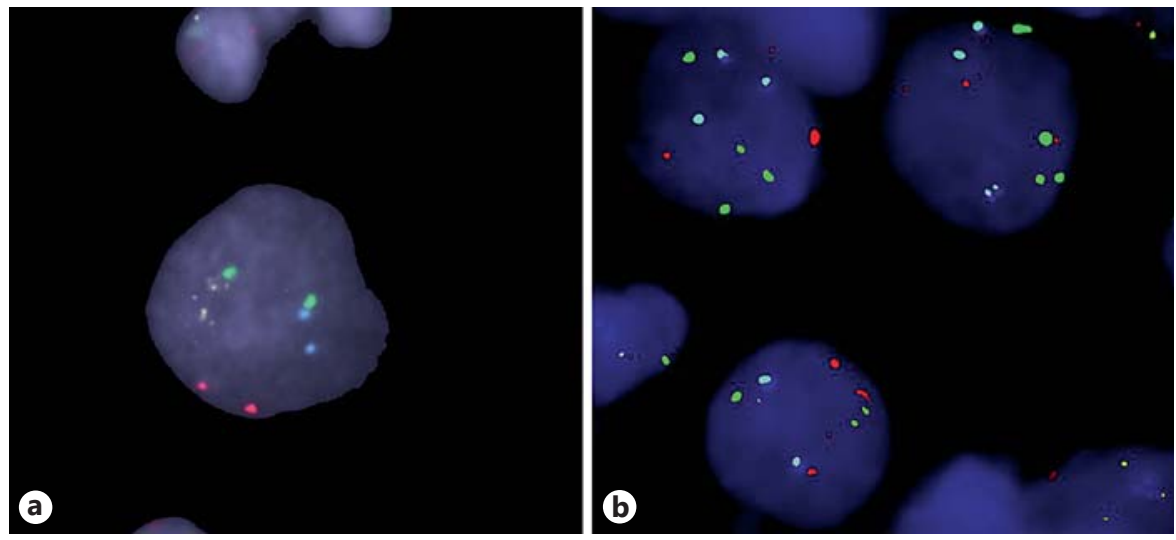

metastatic carcinomas and include TTF- 1 in most lung adenocarcinomas, PAX-8 in ovarian and renal carcinomas, CDX2 in colorectal carcinoma and estrogen receptor and GATA3 in breast carcinoma.

An often more challenging task is to differentiate between reactive, benign mesothelial cells and MM. ICC and IHC are often helpful. The most often recommended epitope is desmin, which is lost early during the malignant transformation of mesothelial cells, but some reactivity may remain in the malignant cell population and the effusion may be a mixture of malignant and benign mesothelial cells. Reactivity to EMA in most cases overrides the weaker reactivity to desmin in the malignant cells, and the use of desmin/EMA double stains is recommended. In some cases there is also a general reactivity to p53, indicating a malignant condition. The use of ploidy analysis by UroVysion ${ }^{\circledR}$ FISH will in most cases indicate malignancy [48].

As with pleural MM, the overwhelming majority of peritoneal MM is epithelioid [34], and the main differential diagnosis for $\mathrm{MM}$ in ascitic fluids is gastrointestinal cancer of pancreatic, gastric or primarily colonic colorectal origin or, in women, primary peritoneal, tubal or ovarian carcinoma, mainly of the serous type.

For further test panels applicable in ICC and IHC for particular differential diagnostic challenges, see the Appendix section of Serous Effusions, edited by Davidson et al. [11].

\section{Medico-Legal Aspects}

Medico-legal implications constitute a significant preanalytical consideration when contemplating the cytological diagnosis of MM. The connection between the le- thal malignancy of MM and its deadly etiological agent asbestos, whose source is usually easily traceable, resulted in a worldwide wave of litigation, which is far from subsiding. The litigation process requires an unequivocal diagnosis based on a variety of specimens, and raises a number of issues for the pathologist who first considers the diagnosis of MM. All cytopathological diagnoses may be scrutinized by expert witnesses, debated by numerous attorneys and end up in a court of law, but this is particularly so with MM. The diagnosis of MM must be made based on solid grounds, including cytomorphology and ancillary techniques such as ICC and IHC, and in most countries a definitive diagnosis of $\mathrm{MM}$ on this basis is currently accepted in everyday medical practice and in courts of law with or without a concurrent histopathological diagnosis.

The long latency period ensures that MM is far from disappearing. Despite severe regulation and banning of the mining and use of asbestos in the USA, Australia, South Africa and Europe, it is expected that the incidence of MM will continue to increase for some time in these countries and also in newly industrialized, less regulated societies throughout the world, where mining and various industrial applications of asbestos occur. Litigation, therefore, will be a fact of life as long as patients and their lawyers succeed in linking MM to asbestos exposure in court cases.

\section{Conclusions}

Although the sensitivity of cytology for the diagnosis of mesothelioma may vary with the experience of cytopathologists, its accuracy is unquestioned when following these guidelines. MM can thus be specifically diagnosed
14

Acta Cytologica 2015;59:2-16 DOI: $10.1159 / 000377697$
Hjerpe et al. 
with a positive predictive value of $100 \%$ based on the examination of an effusion, using cytology with appropriate ancillary techniques [5]. The diagnosis can in this way be obtained earlier and with less invasive techniques. In addition, a positive pleural cytology is a reliable indicator of visceral pleural invasion [49].

There is agreement regarding: (1) the means to distinguish benign reactive mesothelial proliferations from MM; (2) the key cytologic features of pleural and peritoneal MM cells in effusions; (3) the use of ancillary techniques in the diagnosis and differential diagnosis of $M M$, and (4) the use, where necessary, of electron microscopy on effusion cell pellets.

The accurate diagnosis can in the majority of cases be obtained earlier, making further invasive diagnostic measures unnecessary, but it is recognized that sarcomatoid or 'nonepithelioid' MM are not detected in this way, and MM in effusions with a heavy admixture of blood and inflammatory cells pose diagnostic difficulties. These guidelines are meant to be a practical reference for the pathologist trained in cytopathology, and for the physician managing patients with possible MM.

\section{General Recommendations}

The cytological diagnosis of MM in effusions should fulfil one of the following criteria:

- Indisputable malignant cells on cytomorphological criteria which demonstrate a mesothelial phenotype, which should be verified by ancillary techniques;

- Cytomorphological features which are not unequivocally malignant, but ancillary techniques confirm malignancy and a mesothelial phenotype.

When evaluated in clinical practice, these two options can make the specific diagnosis of MM with a high degree of sensitivity and accuracy [5], while noting that the diagnosis of sarcomatoid MM can rarely be established by effusion cytology.

\section{Acknowledgments}

We are grateful to the respective publishers, Karger (Acta Cytologica) and Wiley (Diagnostic Cytopathology), for agreeing to the special arrangement allowing these IMIG guidelines to be published simultaneous by the following two international cytology organizations: the International Academy of Cytology and the Papanicolaou Society of Cytopathology.

\section{References}

1 Husain AN, Colby TV, et al: Guidelines for pathologic diagnosis of malignant mesothelioma: a consensus statement from the International Mesothelioma Interest Group. Arch Pathol Lab Med 2009;133:1317-1331.

2 Husain AN, Colby T, et al: Guidelines for pathologic diagnosis of malignant mesothelioma: 2012 update of the consensus statement from the International Mesothelioma Interest Group. Arch Pathol Lab Med 2013; 137:647-667.

3 Rakha EA, Patil S, et al: The sensitivity of cytologic evaluation of pleural fluid in the diagnosis of malignant mesothelioma. Diagn $\mathrm{Cy}$ topathol 2010;38:874-879.

4 Paintal A, Raparia K, et al: The diagnosis of malignant mesothelioma in effusion cytology: a reappraisal and results of a multi-institution survey. Cancer Cytopathol 2013;121:703707.

5 Segal A, Sterrett GF, et al: A diagnosis of malignant pleural mesothelioma can be made by effusion cytology: results of a 20 year audit. Pathology 2013;45:44-48.

6 Sheaff M: Should cytology be an acceptable means of diagnosing malignant mesothelioma? Cytopathology 2011;22:3-4.

7 Henderson DW, Reid G, et al: Challenges and controversies in the diagnosis of mesothelioma. Part 1. Cytology-only diagnosis, biopsies, immunohistochemistry, discrimination between mesothelioma and reactive mesothelial hyperplasia, and biomarkers. J Clin Pathol 2013;66:847-853.

8 Whitaker D: The cytology of malignant mesothelioma. Cytopathology 2000;11:139-151.

9 Bedrossian CWM: Malignant Effusions: A Multimodal Approach to Cytologic Diagnosis. New York, Igaku Shoin, 1994.

10 Tao L: Cytopathology of Malignant Effusions. Chicago, ASCP Press, 1996.

11 Davidson B, Firat P, et al (eds): Serous Effusions: Etiology, Diagnosis, Prognosis, and Therapy. London/New York, Springer, 2012.

12 Michael CW, Chhieng D, Bedrossian CWM (eds): Cytohistology of the Serous Membranes. New York, Cambridge University Press, 2015.

13 Zellos LS, Sugarbaker DJ: Multimodality treatment of diffuse malignant pleural mesothelioma. Semin Oncol 2002;29:41-50.

14 Ismail-Khan R, Robinson LA, et al: Malignant pleural mesothelioma: a comprehensive review. Cancer Control 2006;13:255-263.

15 Michael CW, Bedrossian CC: The implementation of liquid-based cytology for lung and pleural-based diseases. Acta Cytol 2014;58: 563-573.

16 Widehn S, Kindblom LG: A rapid and simple method for electron microscopy of paraffin- embedded tissue. Ultrastruct Pathol 1988;12. 131-136.

17 Kwee WS, Veldhuizen RW, et al: Quantitative and qualitative differences between benign and malignant mesothelial cells in pleural fluid. Acta Cytol 1982;26:401-406.

18 Matsumoto S, Nabeshima K, et al: Morphology of 9p21 homozygous deletion-positive pleural mesothelioma cells analyzed using fluorescence in situ hybridization and virtual microscope system in effusion cytology. Cancer Cytopathol 2013;121:415-422.

19 Kho-Duffin J, Tao LC, et al: Cytologic diagnosis of malignant mesothelioma, with particular emphasis on the epithelial noncohesive cell type. Diagn Cytopathol 1999;20:5762.

20 Boon ME, van Velzen D, et al: Analysis of number, size and distribution patterns of lipid vacuoles in benign and malignant mesothelial cells. Anal Quant Cytol 1984;6:221-226.

21 Boon ME, Veldhuizen RW, et al: Qualitative distinctive differences between the vacuoles of mesothelioma cells and of cells from metastatic carcinoma exfoliated in pleural fluid. Acta Cytol 1984;28:443-449.

22 Leong AS, Parkinson R, et al: 'Thick' cell membranes revealed by immunocytochemical staining: a clue to the diagnosis of mesothelioma. Diagn Cytopathol 1990;6:9-13. 
23 Ordonez NG, Sahin AA: Diagnostic utility of immunohistochemistry in distinguishing between epithelioid pleural mesotheliomas and breast carcinomas: a comparative study. Hum Pathol 2014;45:1529-1540.

24 Dejmek A, Hjerpe A: Carcinoembryonic antigen-like reactivity in malignant mesothelioma: a comparison between different commercially available antibodies. Cancer 1994;73: 464-469.

25 Dejmek A, Hjerpe A: Reactivity of six antibodies in effusions of mesothelioma, adenocarcinoma and mesotheliosis: stepwise logistic regression analysis. Cytopathology 2000 11:8-17.

26 Saad RS, Cho P, et al: The value of epithelial membrane antigen expression in separating benign mesothelial proliferation from malignant mesothelioma: a comparative study. $\mathrm{Di}$ agn Cytopathol 2005;32:156-159.

27 Sato A, Torii I, et al: Immunocytochemistry of CD146 is useful to discriminate between malignant pleural mesothelioma and reactive mesothelium. Mod Pathol 2010;23:14581466.

28 Husain AN, Mirza MK, et al: How useful is GLUT-1 in differentiating mesothelial hyperplasia and fibrosing pleuritis from epithelioid and sarcomatoid mesotheliomas? An international collaborative study. Lung Cancer 2014; 83:324-328.

29 Minato $\mathrm{H}$, Kurose $\mathrm{N}$, et al: Comparative immunohistochemical analysis of IMP3, GLUT1, EMA, CD146, and desmin for distinguishing malignant mesothelioma from reactive mesothelial cells. Am J Clin Pathol 2014 141:85-93.

30 Hasteh F, Lin GY, et al: The use of immunohistochemistry to distinguish reactive mesothelial cells from malignant mesothelioma in cytologic effusions. Cancer Cytopathol 2010; 118:90-96.
31 Churg A, Allen T, et al: Well-differentiated papillary mesothelioma with invasive foci. Am J Surg Pathol 2014;38:990-998.

32 Washimi K, Yokose T, et al: Well-differentiated papillary mesothelioma, possibly giving rise to diffuse malignant mesothelioma: a case report. Pathol Int 2013;63:220-225.

33 Davidson B: The diagnostic and molecular characteristics of malignant mesothelioma and ovarian/peritoneal serous carcinoma. Cytopathology 2011;22:5-21.

34 Baker PM, Clement PB, et al: Malignant peritoneal mesothelioma in women: a study of 75 cases with emphasis on their morphologic spectrum and differential diagnosis. Am J Clin Pathol 2005;123:724-737.

35 Robinson BW, Creaney J, et al: Mesothelin family proteins and diagnosis of mesothelioma. Lancet 2003;362:1612-1616.

36 Creaney J, Yeoman D, et al: Soluble mesothelin in effusions: a useful tool for the diagnosis of malignant mesothelioma. Thorax 2007;62: 569-576.

37 Pass HI, Wali A, et al: Soluble mesothelin-related peptide level elevation in mesothelioma serum and pleural effusions. Ann Thorac Surg 2008;85:265-272.

38 Creaney J, Dick IM, et al: Pleural effusion hyaluronic acid as a prognostic marker in pleural malignant mesothelioma. Lung Cancer 2013;82:491-498

39 Fujimoto N, Gemba K, et al: Hyaluronic acid in the pleural fluid of patients with malignant pleural mesothelioma. Respir Investig 2013 51:92-97.

40 Mundt F, Nilsonne G, et al: Hyaluronan and $\mathrm{N}$-ERC/mesothelin as key biomarkers in a specific two-step model to predict pleural malignant mesothelioma. PLoS One 2013; 8:e72030.
41 Benjamin H, Lebanony, et al: A diagnostic assay based on microRNA expression accurately identifies malignant pleural mesothelioma. J Mol Diagn 2010;12:771-779.

42 Bovin N, Obukhova P, et al: Repertoire of human natural anti-glycan immunoglobulins. Do we have auto-antibodies? Biochim Biophys Acta 2012;1820:1373-1382.

43 Pass HI, Levin SM, et al: Fibulin-3 as a blood and effusion biomarker for pleural mesothelioma. N Engl J Med 2012;367:1417-1427.

44 Nurminen M, Dejmek A, et al: Clinical utility of liquid-chromatographic analysis of effusions for hyaluronate content. Clin Chem 1994;40:777-780.

45 Wan C, Shen YC, et al: Diagnostic value of fluorescence in situ hybridization assay in malignant mesothelioma: a meta-analysis. Asian Pac J Cancer Prev 2012;13:4745-4749.

46 Adell E, Dejmek A: Telomerase activity analyzed with trap in situ provides additional information in effusions remaining equivocal after immunocytochemistry and hyaluronan analysis. Diagn Cytopathol 2014;42:10511057.

47 Zendehrokh N, Dejmek A: Telomere repeat amplification protocol (TRAP) in situ reveals telomerase activity in three cell types in effusions: malignant cells, proliferative mesothelial cells, and lymphocytes. Mod Pathol 2005; 18:189-196.

48 Flores-Staino C, Darai-Ramqvist E, et al: Adaptation of a commercial fluorescent in situ hybridization test to the diagnosis of malignant cells in effusions. Lung Cancer 2010;68: $39-43$.

49 Pinelli V, Laroumagne S, et al: Pleural fluid cytological yield and visceral pleural invasion in patients with epithelioid malignant pleural mesothelioma. J Thorac Oncol 2012;7:595598 


\section{Erratum}

In the article by Hjerpe et al., entitled 'Guidelines for the cytopathologic diagnosis of epithelioid and mixed-type malignant mesothelioma. Complementary statement from the International Mesothelioma Interest Group, also endorsed by the International Academy of Cytology and the Papanicolaou Society of Cytopathology' [Acta Cytologica 2015;59: 2-16, DOI: 10.1159/000377697], Pap stain applies for panels a, c, e, g and not a, c, e, f as stated in the legend to figure 3. 